\title{
Existence of Electronic Excitation Enhanced Crystallization in GeSb Amorphous Thin Films upon Ultrashort Laser Pulse Irradiation
}

\author{
J. Solis,${ }^{1, *}$ C. N. Afonso, ${ }^{1}$ S. C. W. Hyde,${ }^{2}$ N. P. Barry, ${ }^{2}$ and P. M. W. French ${ }^{2}$ \\ ${ }^{1}$ Instituto de Optica, CSIC. C/Serrano 121, 28006-Madrid, Spain \\ ${ }^{2}$ Femtosecond Optics Group, Optics Department, Imperial College of Science and Technology, Prince Consort Road, \\ London SW7-2BZ, United Kingdom
}

(Received 3 May 1995; revised manuscript received 29 November 1995)

\begin{abstract}
The energy density crystallization threshold of amorphous GeSb films has been studied for the first time as a function of the laser pulse duration in the range from 170 fs to $8 \mathrm{~ns}$. The results obtained provide evidence of the existence of enhanced crystallization upon irradiation with pulses shorter than $800 \mathrm{fs}$, which is most likely related to electronic excitation effects.
\end{abstract}

PACS numbers: 61.80.Ba, 42.65.Re, 61.43.Dq, 64.70.Kb

The study of the interaction of ultrashort laser pulses with solids has raised some important questions concerning the nature of the transformations occurring at the material surface in the presence of very strong levels of electronic excitation. The existence of electronic excitation induced phase transitions has been already demonstrated in several materials ( $\mathrm{Si}$ [1], GaAs [2-4], graphite [5]). In the case of crystalline semiconductors, excitation with pulses shorter than the longitudinal optical phonon emission time [6] may lead to a structural instability of the lattice, leading to the formation of a metastable transient phase with (semi)metallic character. It is not clear, however, whether the presence of such transient strong electronic excitation and/or transient phases may influence the final state or structure induced since, once the energy of the excited carriers has been transferred to the lattice, the structural transformation path is thermal in nature. Different nonthermal mechanisms which could lead to enhanced crystallization and to crystal damage recovery upon pulsed laser irradiation were proposed during the early 1980s [7,8]. More recently, both photonic effects and electron-hole plasma induced network softening have been invoked to explain structural changes induced in amorphous $\mathrm{Si}$ and $\mathrm{Ge}$ upon ns and ps laser pulse irradiation [9-11].

High Sb-content $\mathrm{GeSb}$ amorphous and crystalline thin films have been shown to both crystallize and amorphize upon irradiation with subnanosecond laser pulses. Both processes show optical contrast [12] making this material a potential candidate for applications in ultrashort laser pulse driven phase change optical recording [13,14]. These characteristics also make this material interesting for the study of possible high electronic excitation induced and enhanced crystallization processes. The aim of this work is to elucidate whether the high electronic excitation, induced by irradiation with subnanosecond laser pulses, can induce or enhance the crystallization process of the amorphous phase. Such an enhancement process should lead to appreciable changes in the minimum energy density required for crystallization as the laser pulse duration changes, according to the differences in the excited carrier populations induced. We have performed what we believe to be the first experiment to determine the minimum energy density required for crystallization of an amorphous phase upon irradiation with laser pulses whose durations extend over 3 orders of magnitude, covering the range $170 \mathrm{fs}$ to $8 \mathrm{~ns}$.

The samples used in the experiment were $50 \mathrm{~nm}$ thick, $\mathrm{Ge}_{0.07} \mathrm{Sb}_{0.93}$ films grown at room temperature in a multitarget magnetron dc sputtering system from $\mathrm{Ge}$ and $\mathrm{Sb}$ (99.999\% purity) targets onto glass, carbon-coated mica, and $\mathrm{Si}(100)$ substrates. In order to improve the sensitivity of the material and to enhance the crystallization rate upon pulsed laser irradiation, two different kinds of samples were grown: (a) fully amorphous layers with the composition $\mathrm{Ge}_{0.07} \mathrm{Sb}_{0.93}$ and (2) bilayered films formed by an amorphous surface layer of similar composition and an underlayer slightly richer in $\mathrm{Sb}$ and therefore polycrystalline. Since the bilayered films showed smaller transformation times and improved sensitivity upon pulsed laser irradiation with $10 \mathrm{ps}$ laser pulses at $583 \mathrm{~nm}$, they were chosen for the present work. The samples on mica and $\mathrm{Si}$ were used, respectively, to prepare planar and cross-section transmission electron microscopy (TEM) specimens in order to characterize film structure and composition. The latter was determined by energy dispersive $\mathrm{x}$-ray spectroscopy on planar TEM specimens.

An ultrashort CPA laser pulse source (described in Ref. [15]) was used to irradiate the samples on glass. The experimental configuration is shown in Fig. 1. A commercial fs $\mathrm{Ti}_{2} \mathrm{Al}_{2} \mathrm{O}_{3}$ oscillator providing 150 fs duration pulses at a repetition rate of $82 \mathrm{MHz}$ at $830 \mathrm{~nm}$ was used to seed an $\mathrm{Ar}^{+}$ion pumped Cr-LiSAF solid state regenerative amplifier. Before seeding the amplifier, the oscillator pulses were stretched to approximately 300 ps with a diffraction grating pulse stretcher. After amplification the pulses were recompressed with a grating pair compressor. The typical output of the laser system consisted of a $10 \mathrm{kHz}$ repetition rate pulse train with energies per pulse in the order of $5 \mu \mathrm{J}$. A second Pockels cell, synchronized to the one in the regenerative amplifier, was used 


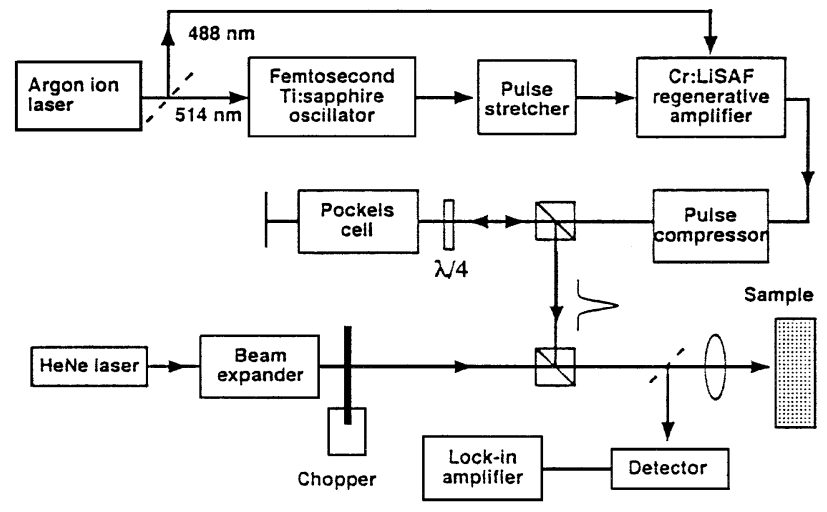

FIG. 1. Experimental setup for pulsed laser irradiation. The laser pulse source operates at $830 \mathrm{~nm}$ and may produce pulses with durations ranging from 400 fs to $8 \mathrm{~ns}$. The reflectivity of the sample at $633 \mathrm{~nm}$ is monitored before and after irradiation.

to switch out pulses from the pulse train in order to perform single-shot irradiation experiments, and to suppress the low energy background signal of unamplified pulses at $82 \mathrm{MHz}$ in the amplifier output. By adjusting the stretching and compression elements in the CPA system, the output pulse duration could be varied from hundreds of fs to hundreds of ps. Additional irradiation experiments with ns pulses were performed by blocking the seed pulses to the amplifier, which then acted as a conventional $Q$-switch ns pulsed laser.

The laser beam was focused on the sample surface to a spot size of the order of $15 \mu \mathrm{m}$ by means of a NA 0.4 , $10 \times$ microscope objective. A set of energy calibrated fast detectors allowed both the pulse energy to be monitored and the energy deposited in the sample to be determined. Since crystallization is evidenced by significant changes in the reflectivity of the surface, a cw $\mathrm{HeNe}(633 \mathrm{~nm})$ laser beam arranged to be collinear with the irradiation beam was used to monitor the reflectivity of the surface before and after irradiation. The HeNe laser beam was expanded before overlapping in order to focus it to a size of $\approx 2 \mu \mathrm{m}$ at the sample site and is therefore probing a nearly homogeneously irradiated region. After each single shot irradiation the sample was moved to a fresh region.

Figure 2 shows the evolution of the reflectivity changes induced upon irradiation as a function of the pulse energy for a fixed pulse length. It can be seen that for very low pulse energies the reflectivity of the surface remains unchanged. There is a transformation threshold above which a positive reflectivity change is observed. This reflectivity increase has been previously reported upon irradiation of similar composition films with ns [13], subnanosecond, and subpicosecond laser pulses [12,14] and is related to the crystallization of the amorphous phase. The reflectivity change increases with the pulse energy as the depth of the induced crystalline phase increases, until a saturation value of approximately a $32 \%$ reflectivity change is reached. For higher energies,

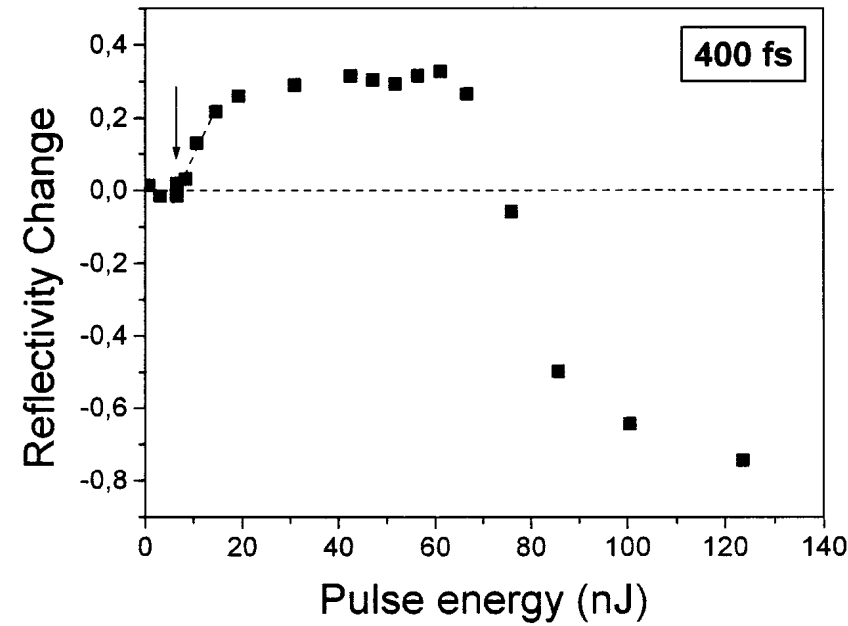

FIG. 2. Evolution of the reflectivity of the GeSb films after irradiation with $400 \mathrm{fs}$ laser pulses versus the pulse energy measured at the sample site. The induced reflectivity changes have been normalized to the initial reflectivity of the sample. The arrow denotes the pulse energy threshold for crystallization.

surface ablation occurs, leading to a sharp decrease of reflectivity. Similar curves for the evolution of the reflectivity versus the pulse energy have been obtained for pulse lengths ranging from $400 \mathrm{fs}$ to $8 \mathrm{~ns}$. The minimum energy required for surface crystallization has been determined in these curves as the intersection of the linear extrapolation of the leading edge of the curve with the horizontal axis (see the arrow in Fig. 2).

Since the pulses of different durations are produced in the CPA system by adjusting the alignment of the stretching and compression elements, some differences in the beam spatial profile may occur for different output pulse durations. These differences could lead to apparent changes in the crystallization threshold that are related to changes in the spatial distribution of energy in the sample for different pulse lengths. In order to eliminate such possible artifacts, the absolute energy density crystallization threshold was carefully determined as follows. For a Gaussian beam, the area over which the local energy density is above a given constant value scales linearly with the logarithm of the pulse energy. The slope of such a plot is $M=\pi r^{2}$, where $r$ is the $1 / e$ beam radius of the distribution. The maximum of the local energy density distribution is then the pulse energy divided by $M$. For each pulse length, the area of the crystallized surface was measured and plotted as a function of the logarithm of the pulse energy, as can be seen in Fig. 3. The low energy region of the curve was then fitted by a straight line and the value of $M$ was determined. The variation of the energy density crystallization threshold, calculated as the minimum energy required for crystallization divided by $M$, is plotted in Fig. 4 as a function of the pulse length. The error bars included correspond to the cumulative effect of errors in the determination of the energy deposited at the sample 


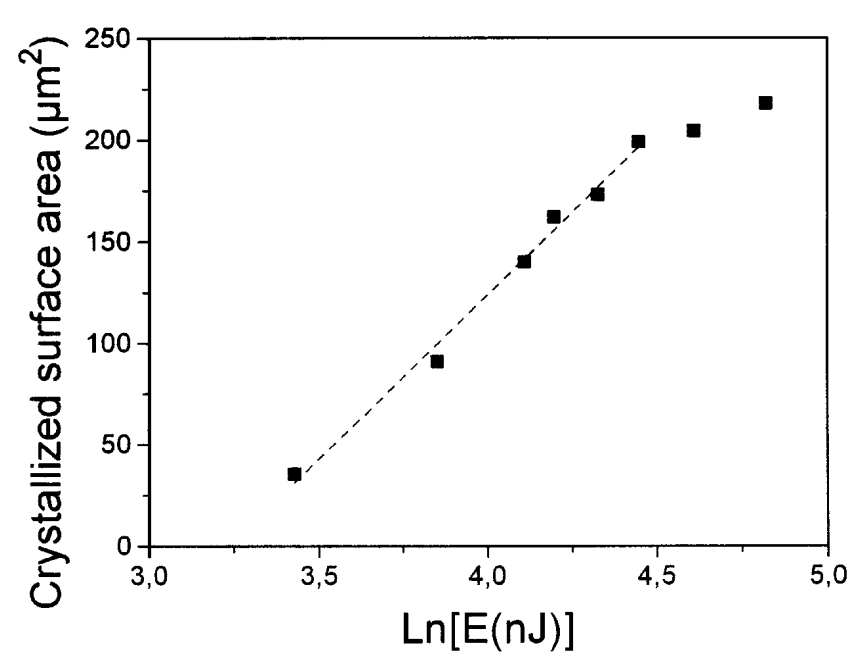

FIG. 3. Area of the crystallized surface upon irradiation with $400 \mathrm{fs}$ laser pulses as a function of the logarithm of the pulse energy. The dashed line corresponds to a linear fit of the low energy region.

$(\approx 5 \%)$, crystallized surface area measurements $(<10 \%)$, and linear fitting of the crystallized surface area versus the logarithm of the pulse energy $(<15 \%)$. It is clear that a significant increase in the threshold occurs when the irradiation pulse is in the ns time scale. For subnanosecond pulses, the energy density threshold remains approximately constant within the experiment error and only for the shortest pulse length (400 fs) can a clear decrease in the threshold be seen.

The origin of the increase in the energy density crystallization threshold for pulses in the ns time scale is related to the existence of heat transfer to the substrate, while the laser pulse is still being absorbed by the sample. For a light skin penetration depth smaller than the film thickness and assuming a carrier-network thermalization

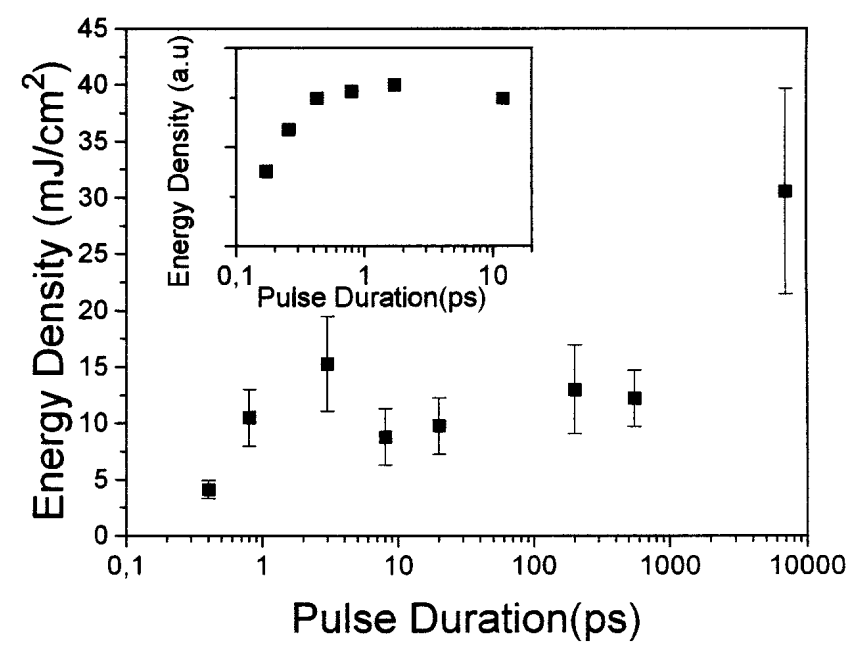

FIG. 4. Energy density crystallization threshold of the GeSb films versus pulse duration. The inset shows the evolution obtained in an additional set of measurements with pulse durations in the range from 170 fs to 1 ps. time much shorter than the ns pulse length, the time required for heat to diffuse to the substrate can be estimated by assuming that the thermal diffusion length $\lambda=\sqrt{D t}$ equals the film thickness $(50 \mathrm{~nm})$. In this expression $D$ is the film thermal diffusitivity $(D=$ $k / \rho C_{p}, k$ the thermal conductivity, $\rho$ the density, and $C_{p}$ the specific heat) and $t$ is the time elapsed from the pulse onset. Since the Ge content of the film is very low, and since it is known that $\mathrm{Ge}$ incorporates in the crystalline lattice of Sb upon crystallization with short and ultrashort laser pulses [13], we have used the thermal constants of bulk crystalline $\mathrm{Sb}\left(D=0.16 \mathrm{~cm}^{2} \mathrm{~s}^{-1}[16]\right)$ in order to estimate a value of $t \approx 200 \mathrm{ps}$. This value is likely to be an underestimation since it is well known that the thermal conductivity of amorphous phases can be much smaller than that of the corresponding crystalline material [17-19]. With a decreased thermal conductivity for the amorphous phase, a value for $t$ in the ns range is to be expected. Obviously, for a pulse duration of the order of the thermal diffusion time or longer, heat is transferred to the substrate, while the pulse is being absorbed by the film. Therefore the peak temperatures reached are smaller with a subsequent increase in the crystallization threshold.

The absence of significant changes in the threshold value for pulse durations in the $800 \mathrm{fs}-550 \mathrm{ps}$ interval and the decrease observed at 400 fs clearly demonstrates that the enhanced crystallization can only occur for pulses shorter than $800 \mathrm{fs}$. This result also suggests that the time for energy transfer from excited carriers to the lattice has to be shorter than $800 \mathrm{fs}$. This value is reasonable since it is generally accepted that the energy transfer mechanisms from excited carriers to the network in amorphous materials can be very efficient (in the 1 ps range or even shorter) due to the relaxation of the $k$-vector selection rule [20] and the presence of other phenomena like trapping [21].

The presence of a nonlinear absorption mechanism for large peak intensities could lead to an increase in the amount of pulse energy absorbed for the shortest pulses, giving rise to the observed decrease in the energy density crystallization threshold at $400 \mathrm{fs}$. This effect was investigated by measuring the film reflectivity at $830 \mathrm{~nm}$ for pulse durations ranging from $100 \mathrm{fs}$ to approximately 100 ps at several fluences below the transformation threshold. The observed reflectivity value remains constant and equal to $0.48 \pm 0.02$ in all cases, which allows us to discard nonlinear optical effects as responsible for the decrease observed for 400 fs laser pulses.

In order to further investigate the mechanism change occurring for pulse durations shorter than $800 \mathrm{fs}$, an additional set of measurements with pulse durations ranging from approximately $10 \mathrm{ps}$ to $100 \mathrm{fs}$ was performed. Since the initial experiment configuration does not allow us to achieve pulse durations shorter than $400 \mathrm{fs}$, the second set was performed using a similar experimental setup but without stretching the pulses before amplification and compressing them at the output of the second Pockels cell 
(see Fig. 1). This leads to an improved beam quality and reproducibility but also to a different (and bigger) probe to pump beam size ratio at the sample site that does not guarantee that the region probed by the $\mathrm{HeNe}$ beam is homogeneously irradiated. Therefore the energy density threshold values obtained are not absolute. The result of this additional set of measurements can be seen as an inset in Fig. 4. The evolution of the crystallization threshold between $170 \mathrm{fs}$ and 12 ps confirms further that the threshold remains constant for pulses longer than 800 fs and evidences that there is a decrease as the pulse duration is reduced down to $170 \mathrm{fs}$. This provides additional evidence of the existence of enhanced crystallization upon irradiation of the amorphous phase with ultrashort laser pulses. Since nonlinear optical effects have been discarded as responsible for this behavior, the crystallization enhancement has to be most likely related to electronic excitation effects.

In conclusion, the energy density threshold for optically induced structural transformations (crystallization) in GeSb amorphous films has been studied for the first time for pulse durations ranging from 170 fs to $8 \mathrm{~ns}$. The results evidence the existence of appreciable heat flow during the pulse absorption for ns pulses and demonstrate that enhanced crystallization occurs for pulses shorter than $800 \mathrm{fs}$, most likely related to electronic excitation effects. The absence of changes in the energy density crystallization threshold for subnanosecond pulses longer than 800 fs suggests a carrier-lattice thermalization time in the hundreds of fs range.

This work has been partially supported by CICYT (Spain) under the TIC93-0125 project and by the Royal Society and the EPRSC (UK). We are grateful to the British Council/CSIC Joint Research Program for travelling finances. S.C.W. Hyde acknowledges an EPRSC Cooperative award studentship supported by Kodak Ltd. We would like to thank J. M. Ballesteros (I. Optica, CSIC, Spain) for his help in analyzing the optical microscopy data.

\footnotetext{
*Electronic address: IODJS37@pinar1.csic.es
}

[1] H.W.K. Tom, G.D. Aumillier, and C.H. Brito-Cruz, Phys. Rev. Lett. 60, 1438 (1988).

[2] Y. Siegal, E. N. Glezer, and E. Mazur, Phys. Rev. B 49, 16403 (1994).

[3] S. V. Govorkov, Th. Schröder, I. L. Shumay, and P. Heist, Phys. Rev. B 46, 6864 (1992).

[4] P. Saeta, J.-K. Wang, Y. Siegal, N. Bloembergen, and N. Mazur, Phys. Rev. Lett. 67, 1023 (1991).

[5] D. H. Reitze, X. Wang, H. Ahn, and M. C. Downer, Phys. Rev. B 40, 11986 (1989).

[6] J. A. Kash, J. C. Tsang, and J. M. Hvam, Phys. Rev. Lett. 54, 2151 (1985).

[7] J. A. Van Vechten, in Laser and Electron Beam Processing of Semiconductors, edited by C. W. White and P.S. Peercy (Academic Press, New York, 1980).

[8] J. A. Van Vechten, R. Tsu, and F. W. Saris, Phys. Lett. 74A, 422 (1979).

[9] W. Sinke, T. Warabisako, M. Miyao, T. Tokuyama, S. Roorda, and F. W. Saris, J. Non-Cryst. Solids 99, 308 (1988).

[10] M. G. Grimaldi, P. Baeri, and M. A. Malvezzi, Phys. Rev. B 44, 1546 (1991).

[11] J. Marfaing and W. Marine, Phase Trans. 14, 225 (1989).

[12] C. N. Afonso, J. Solis, F. Catalina, and C. Kalpouzos, Appl. Phys. Lett. 60, 3123 (1992).

[13] J. Solis, C. N. Afonso, J.F. Trull, and M.C. Morilla, J. Appl. Phys. 75, 7788 (1994).

[14] C. N. Afonso, M. C. Morilla, J. Solis, N. H. Rizvi, M. A. Ollacarizqueta, and F. Catalina, Mater. Sci. Eng. A173, 343 (1993).

[15] S. C. W. Hyde, N. P. Barry, R. Mellish, P. M. W. French, J. R. Taylor, C. J. van der Poel, and A. Valster, Opt. Lett. 20, 160 (1995).

[16] Smithells Metals Reference Book, edited by Eric A. Brandes (Butterworths, London, 1983), 6th Ed.

[17] W. Szysko, F. Vega, and C. N. Afonso, Appl. Phys. A61, 141 (1995).

[18] D. G. Cahill, H. E. Fisher, T. Klistner, E. T. Schwartz, and R. O. Pohl, J. Vac. Sci. Technol. A 7, 1259 (1989).

[19] T. Q. Qui and C.L. Lien, Trans. ASME 115, 842 (1993).

[20] D. Hulin, A. Mourchid, P. M. Fauchet, W. L. Nighan, Jr., and R. Vanderhagen, J. Non-Cryst. Solids 137\&138, 527 (1991).

[21] M. Wraback, J. Tauc, D. Pang, W. Paul, J.-K. Lee, and E. A. Schiff, J. Non-Cryst. Solids 137\&138, 531 (1991). 